\title{
Nuclear IGF-1R predicts chemotherapy and targeted therapy resistance in metastatic colorectal cancer
}

Jordi Codony-Servat 1,2,19, Miriam Cuatrecasas 3,19, Elena Asensio ${ }^{4}$, Carla Montironi ${ }^{3}$, Anna Martínez-Cardús², Mercedes Marín-Aguilera ${ }^{1}$, Carlos Horndler ${ }^{5}$, Eva Martínez-Balibrea ${ }^{2}$, Michele Rubini ${ }^{6}$, Pedro Jares $^{3}$, Oscar Reig ${ }^{1}$, Iván Victoria ${ }^{1}$, Lydia Gaba ${ }^{1}$, Marta Martín-Richard ${ }^{7}$, Vicente Alonso ${ }^{8}$, Pilar Escudero ${ }^{9}$, Carlos Fernández-Martos ${ }^{10}$, Jaime Feliu ${ }^{11}$, Jose Carlos Méndez ${ }^{12}$, Miguel Méndez ${ }^{13}$, Javier Gallego ${ }^{14}$, Antonieta Salud ${ }^{15}$, Federico Rojo ${ }^{16}$, Antoni Castells ${ }^{4}$, Aleix Prat ${ }^{1}$, Rafael Rosell ${ }^{2}$, Xabier García-Albéniz ${ }^{17}$, Jordi Camps ${ }^{\star 4,18,20}$ and Joan Maurel ${ }^{\star, 1,20}$

${ }^{1}$ Medical Oncology Department, Hospital Clínic, Translational Genomics and Targeted Therapeutics in Solid Tumors Group, IDIBAPS, Universitat de Barcelona, c/ Villarroel, 170, Barcelona 08036, Spain; ${ }^{2}$ Medical Oncology Service, Institut Català d'Oncologia-Hospital Germans Trias i Pujol, Crta. de Canyet, s/n, Badalona 08916, Spain; ${ }^{3}$ Pathology Department, Hospital Clínic and Tumour Bank-Biobank, IDIBAPS, Universitat de Barcelona, c/ Villarroel, 170, Barcelona 08036, Spain; ${ }^{4}$ Gastrointestinal and Pancreatic Oncology Group, Institut D'Investigacions Biomèdiques August Pi i Sunyer (IDIBAPS), Centro de Investigación Biomédica en Red de Enfermedades Hepáticas y Digestivas (CIBERehd), c/ Rosselló, 149153, Barcelona 08036, Spain; ${ }^{5}$ Pathology Service, Hospital Miguel Servet, Paseo Isabel la Católica, 1-3, Zaragoza 50009, Spain; ${ }^{6}$ Department of Experimental and Diagnostic Medicine, University of Ferrara, Via Savonarola, 9, Ferrara FE 44121, Italy; ${ }^{7}$ Department of Oncology, Hospital Sant Pau, c/ Sant Quintí, 89, Barcelona 08026, Spain; ${ }^{8}$ Department of Oncology; Hospital Miguel Servet, Paseo Isabel la Católica, 1-3, Zaragoza 50009, Spain; ${ }^{9}$ Department of Oncology, Hospital Clínico Universitatio Lozano Blesa, Av. San Juan Bosco, 15, Zaragoza 50009, Spain; ${ }^{10}$ Department of Medical Oncology, Instituto Valenciano de Oncología, c/ Professor Beltrán Báguena, 8, Valencia 46009, Spain; ${ }^{11}$ Department of Oncology, Hospital La Paz, CIBERONC, Pso. de la Castellana, 261, Madrid 28046, Spain; ${ }^{12}$ Department of Oncology, Centro Oncológico de Galicia, c/ Doctor Camilo Veiras, 1, A Coruña 15009, Spain; ${ }^{13}$ Department of Oncology, Hospital de Móstoles, Río Júcar s/n, Móstoles 28935, Spain; ${ }^{14}$ Department of Oncology, Hospital de Elche, c/ Almazara, 11, Elche 03203, Spain; ${ }^{15}$ Department of Oncology, Hospital Arnau de Vilanova, Av. Alcalde Rovira Roure, 80, Lleida 25198, Spain; ${ }^{16}$ Pathology Department, Fundación Jiménez Diaz University, Av. Reyes Católicos, 2, Madrid 28040, Spain; ${ }^{17}$ Harvard T.H. Chan School of Public Health, 677 Huntington Ave, Boston, MA 02115, USA and ${ }^{18}$ Unitat de Biologia Cel.lular i Genètica Mèdica, Departament de Biologia Cel·lular, Fisiologia i Immunologia, Universitat Autònoma de Barcelona, Edifici M, Cerdanyola del Vallès 08193, Spain

Background: Although chemotherapy is the cornerstone treatment for patients with metastatic colorectal cancer (mCRC), acquired chemoresistance is common and constitutes the main reason for treatment failure. Monoclonal antibodies against insulin-like growth factor- 1 receptor (IGF-1R) have been tested in pre-treated $\mathrm{mCRC}$ patients, but results have been largely deceiving.

Methods: We analysed time to progression, overall survival, and the mutational status of RAS, BRAF and nuclear p-IGF-1R expression by immunohistochemistry, in 470 metastatic CRC patients. The effect of IGF-1R activation and distribution was also assessed using cellular models of CRC and RNAi for functional validation.

Results: Nuclear IGF-1R increased in metastatic tumours compared to paired untreated primary tumours, and significantly correlated with poor overall survival in $\mathrm{mCRC}$ patients. In vitro, chemo-resistant cell lines presented significantly higher levels of IGF-1R expression within the nuclear compartment, and PIAS3, a protein implicated also in the sumoylation process of intranuclear proteins, contributed to IGF-1R nuclear sequestration, highlighting the essential role of PIAS3 in this process. Intriguingly, we observed that ganitumab, an IGF-1R blocking-antibody used in several clinical trials, and dasatinib, an SRC inhibitor, increased the nuclear localisation of IGF-1R.

Conclusions: Our study demonstrates that IGF-1R nuclear location might lead to chemotherapy and targeted agent resistance.

*Correspondence: Dr J Camps; E-mail: jcamps@clinic.cat or Dr J Maurel; E-mail: jmaurel@clinic.cat

${ }^{19}$ These authors contributed equally to this work.

${ }^{20}$ These authors contributed equally to this work.

Received 15 May 2017; revised 10 July 2017; accepted 24 July 2017; published online 9 November 2017

(C) 2017 Cancer Research UK. All rights reserved 0007-0920/17 
Colorectal cancer (CRC) is one of the most commonly diagnosed cancers and a leading cause of death worldwide (Brenner et al, 2014). Despite currently available first-line treatments with FOLFOX or FOLFIRI and the implementation of targeted therapies such as bevacizumab, panitumumab or cetuximab, the median progression-free survival (PFS) has only improved modestly (9-10 months) and most patients with metastatic colorectal cancer (mCRC) are not cured (Saltz et al, 2008; Van Cutsem et al, 2011). Acquired resistance to chemotherapy and targeted agents is a major obstacle to survival in patients with mCRC.

The type 1 insulin-like growth factor receptor (IGF-1R) is a transmembrane glycoprotein composed of two extracellular units and two cytoplasmic subunits acting as a receptor tyrosine kinase. IGF-1R is overexpressed in many human cancers (Pollak, 2008; Baserga, 2009) and its activation at the plasma membrane mediates key processes in cancer, such as cell growth, cell proliferation, differentiation, apoptosis, angiogenesis, and anchorage independent growth DNA damage repair (Werner and LeRoith, 1996; Baserga, 1999; Girnita et al, 2000). The main signalling pathways involved in IGF-1R are PI3K-AKT and MAPK pathways. IGF-1R has become a target of novel therapeutics, specifically monoclonal antibodies and small molecules targeting inhibition of the tyrosine kinase activity.

Several receptor tyrosine kinases such as EGFR, HER2, HER3, HER4, FGFR1, and FGFR3 are known to undergo nuclear translocation in human cancers (Johnston et al, 1995; Lin et al, 2001; Wang et al, 2004). Upon ligand binding of IGF-1 or IGF-2 to the IGF-1R and constitutional activation of the signalling pathway, the IGF-1R undergoes endocytic internalisation by clathrin-coated vesicles (Salani et al, 2010), and subsequent ubiquitination and degradation by the proteasome (Sehat et al, 2007). It has been recently described that sumoylation of IGF-1R at three evolutionary conserved lysine residues is critical for the nuclear translocation (Sehat et al, 2010; Deng et al, 2011), and once inside the nucleus, IGF-1R would play a role as a transcriptional co-activator of LEF1/TCF complex, increasing the transcription of downstream target genes such as Cyclin D1 and Axin 2 (Warsito et al, 2012). Sumoylation of IGF-1R seems to be required for interaction with $\mathrm{RanBP} 2$, a nuclear pore complex protein crucial for the nucleo-cytoplasmic trafficking, which may favour nuclear IGF-1R accumulation by increasing the stability of the receptor (Packham et al, 2014). Specifically, RANBP2 binds to RANGAP1*SUMO1 and Ubc9, and forms an autonomous machine that disassembles Crm1-dependent nuclear export complexes (Ritterhoff et al, 2016). Recent reports have described the presence of IGF-1R within the nucleus of human tumour renal cells, pre-invasive lesions in the breast and in proliferative non-malignant tissues (Aleksic et al, 2010). According to the same authors, nuclear translocation of IGF-1R is associated with poor prognosis in renal cancer and may influence the efficacy of IGF-1R inhibitory drugs. In BRAF-like colon cancer cells, it has been recently shown that the loss of RANBP2 induces greater cell death upon treatment with vinorelbine due to the malfunctioning of the kinetochore during mitosis (Vecchione et al, 2016). However, whether the internalisation of nuclear IGF-1R plays a role in resistant CRC remains totally elusive.

In addition, despite that some CRC cell lines are sensitive to anti-IGF-1R compounds (both monoclonal antibodies and tyrosine-kinase inhibitors) (Flanigan et al, 2010; Ii et al, 2011), several trials in heavily pre-treated mCRC patients with anti-IGF-1R compounds (Cohn et al, 2013; Van Cutsem et al, 2014) showed only modest efficacy. We therefore sought to understand the potential role that nuclear translocation of IGF-1R might have during the acquisition of chemo-resistance to conventional and targeted therapies in CRC.

\section{MATERIALS AND METHODS}

Patient samples. To evaluate the prognostic role of nuclear p-IGF-1R, we analysed four cohorts of mCRC patients. The SIEMENS-2 cohort consisted of 279 mCRC patients treated with FOLFOX-6 or CAPOX (Alonso-Orduna et al, 2014). The BECOX trial consisted of 68 mCRC patients treated with CAPOX plus bevacizumab (NCT01067053) (Feliu et al, 2014). The PULSE trial consisted of 185 screened mCRC patients of which 78 WT KRAS (exon 2) were included and treated with FOLFOX-6 plus panitumumab (NCT01288339) (Maurel et al, 2016). The POSIBA trial consisted of 212 WT KRAS (exon 2) patients treated either with FOLFOX-6 or FOLFIRI plus biweekly cetuximab (NCT01276379) (Alonso et al, 2016). Since anti-EGFR compounds are approved currently only in all RAS WT patients, we excluded patients from the PULSE and POSIBA trials with mutations in KRAS (exons 3 and 4) and NRAS. We studied the patterns and nuclear expression of $\mathrm{p}$-IGF-1R and its association with $R A S$ and $B R A F$ genotypes in available samples from 563 mCRC patients. We also assessed changes of nuclear p-IGF-1R expression in samples taken after treatment with anti-EGFR $+/-$ irinotecan as second or third line of therapy in 44 patients with paired biopsies in a different cohort of $\mathrm{mCRC}$ patients.

The paired biopsy biomarker and SIEMENS-2 studies were approved by the institutional Ethics Committee of Hospital Clínic Barcelona. The BECOX, PULSE and POSIBA trials were approved by local institutional review boards and ethics committees in accordance with national and international guidelines; all patients signed a written informed consent document.

Cell lines. Human colorectal carcinoma cell lines HT29, DLD-1, HCT116, SW1116, Colo320, SW480, SW403, SW1463 and SW837 were obtained from the American Type Culture Collection (ATCC, Manassas, VA, USA) and cultured according to ATCC conditions. HT29-OxR and DLD-1-OxR are oxaliplatin resistant cell lines generated from HT29 and DLD1 parental cell lines respectively, kindly provided by Dr Albert Abad from Hospital Germans Trias i Pujol (Barcelona, Spain).

Tissue microarray and biopsies. Tissue microarrays were performed from representative tumour areas selected from paraffin blocks of surgical specimens. Each tumour was represented by four $1 \mathrm{~mm}$ diameter cores. Several consecutive $2-3 \mu \mathrm{m}$ cuts of each TMA were performed and stained with haematoxylin and eosin and immunohistochemistry (IHC). In patients for whom surgical specimens were not available, whole sections of the previous diagnostic endoscopic biopsies were used for IHC staining.

Mutational analysis. Mutational analysis of RAS was performed by direct sequencing after COLD-PCR amplification. The BRAF V600E genomic mutation in exon 15 was genotyped by allelic discrimination in genomic DNA using TaqMan technology on a 7300 Real Time PCR System (Applied Biosystems, Foster City, CA, USA).

Cell viability assay. The effect on cell viability of 5-fluorouracil (5FU), oxaliplatin, sorafenib and ganitumab were studied by using the colorimetric method provided by MTS-Cell Titer 96 Aqueous Non-Radioactive Cell Proliferation Assay Kit (Promega, Madison, WI, USA). Briefly, 2000 cells were seeded in $100 \mu$ l drug-free media and incubated for $24 \mathrm{~h}$ before drug treatment in 96-well flatbottomed plates (Nunc, Naperville, IL, USA). Incubations were performed for $72 \mathrm{~h}$ and absorbance generated were measured on an Epoch microplate spectrophotometer (BioTek Instruments, Winooski, VT, USA). Data were derived from three independent experiments. 
Reagents and antibodies. The following reagents and drugs were used in this study: Ganitumab (Amgen, Thousand Oaks, CA, USA), an anti-IGF-1R monoclonal antibody; NVP-AEW541 (Novartis, Basel, Switzerland), a tyrosine kinase inhibitor of IGF-1R; sorafenib (Bayer, Leverkusen, Germany), a multikinase inhibitor (C-Raf, B-Raf, VEGFR2, VEGFR3, PDGFR $\beta$ and C-Kit); cetuximab (provided by the Hospital Clínic Pharmacy Unit), monoclonal antibody against EGFR; TIMP-1 (Chemicon International Inc., Temecula, CA, USA), a matrilysin inhibitor; dasatinib (LC laboratories, Woburn, MA, USA), an inhibitor of BCR-ABL kinase and Src-family kinases; dynasore (Sigma-Aldrich, Saint Louis, MO, USA), an inhibitor of endocytic vesicle formation; curcumin (Sigma-Aldrich); leptomycin B (Selleckchem, Houston, TX, USA), a specific nuclear traffic inhibitor through CRM1; oxaliplatin (Sigma-Aldrich); and 5-FU (provided by the Hospital Clínic Pharmacy Unit).

The primary antibodies used for western blot were tubulin (Sigma-Aldrich), PARP (Roche, Basel, Switzerland), IGF-IR, p-IRS-1, AKT, p-AKT (Ser473), ERK 1/2, p-ERK 1/2, BAX, BCL-2, PIAS3, GAPDH (Cell Signaling Technologies, Danvers, MA, USA), HDAC1 (Rockland, Limerick, PA, USA), and phosphoIGF-1R (Y1316) (kindly provided by Dr M. Rubini from University of Ferrara, Italy). Species-specific HRP-linked antibodies (GE Health Care, Little Chalfont, UK) were used for secondary labelling. For immunofluorescence, we used the following antibodies: mouse anti-E-cadherin (Transduction Lab, San Diego, CA, USA), and rabbit anti-phospho-IGF-1R (Y1316). Alexa Fluor 594 goat anti-rabbit (Life Technologies, Carlsbad, CA, USA) was used as a secondary antibody.

Immunostaining. We used haematoxylin and eosin staining to select representative sections of the tumour for immunostaining. The above-mentioned list of colorectal carcinoma cells lines was cultured, trypsin-based detached and centrifuged. The resulting pellet was formalin-fixed and paraffin-embedded in a cellular block using the plasma-thrombin method. Multiple consecutive $2-3-\mu \mathrm{m}$ thick sections were performed for IHC. One slice was stained with haematoxylin and eosin for morphologic control, while the others were used for IHC stains. Removal of paraffin was performed with xylene and decreasing concentrations of ethanol. Heat antigen retrieval was achieved by incubation of the slides in $\mathrm{pH} 6.0$ buffer citrate at $10{ }^{\circ} \mathrm{C}$ for $20 \mathrm{~min}$. Primary p-IGF-1R antibody was used at $1: 100$, and incubated for $1 \mathrm{~h}$. Secondary antibody, detection and revealed was performed using the Dako (Carpinteria, CA, USA) EnVision K4011 (Dako). Nuclear positivity was recorded using a conventional Olympus BX41 microscope (Olympus, Tokyo, Japan), and defined as negative ( $0 \%$ of cells with nuclear staining), low $(1-19 \%$ of cells with nuclear staining) or high $(\geqslant 20 \%$ of cells with nuclear staining) expression.

For immunofluorescence, paraffin from $3 \mu \mathrm{m}$ primary sample sections was removed and the samples were heated in citrate $\mathrm{pH}$ 6.0 for antigen retrieval. Slides were permeabilised in PBS with triton $0.5 \%$, blocked with normal donkey serum $3 \%$, and incubated with the primary antibody overnight. We performed nuclear staining using TO-PRO-3 stain (1:50000; Life Technologies). Additionally, HT29 and HT29-OxR cell lines were cultured on glass cover slips (Thermo Fisher Scientific, Carlsbad, CA, USA) to 40-50\% confluence. After $24 \mathrm{~h}$ incubation, the cells were fixed with $4 \%$ cold paraformaldehyde and permeabilised in $0.15 \%$ Triton $\mathrm{X}-100$. Fixed cells were blocked and incubated with primary antibodies overnight at $4{ }^{\circ} \mathrm{C}$. Next day, slides were washed and incubated for $1 \mathrm{~h}$ with the corresponding secondary antibody. After washing the slides with PBS, we used Prolong Gold Antifade Reagent with DAPI (Life Technologies) for mounting. Images were acquired with a Leica TCS SP5 confocal microscopy (Leica, Wetzlar, Germany), and were processed and analysed with ImageJ software. Results are expressed as the mean \pm s.e.m., unless otherwise indicated.
Western blot analysis. Cells were cultured in six-well plates or $100 \mathrm{~mm}$ cell culture dishes and total protein extract was obtained. Briefly, for cytoplasmic and nuclear extracts, the Nuclear/Cytosol Fractionation kit (BioVision, San Francisco, CA, USA) was used. Proteins were resolved on SDS-polyacrylamide gels and electrophoretically transferred to polyvinyllidene fluoride membranes. Membranes were blocked in 5\% TBS containing non-fat dried milk and then incubated for $1 \mathrm{~h}$. After this, the membrane was incubated with primary antibody overnight at $4{ }^{\circ} \mathrm{C}$ and then with the corresponding secondary antibody for $2 \mathrm{~h}$. Enhanced chemiluminescence was used according to the manufacturer's instructions (SuperSignal West Dura Chemiluminiscent Substrate, Thermo Fisher Scientific).

Small interfering RNA. Gene silencing was performed by using the SMART pool siRNA against PIAS3 and the corresponding controls (Dharmacon, Lafayette, LA, USA, Thermo Fisher Scientific). Lipid-based transfections were achieved with Lipofectamine 2000 (Life Technologies) according to the manufacturer's protocol. Cells were incubated with the siRNA complex for $72 \mathrm{~h}$ and protein was extracted for assessing transfection efficiency by western blot.

Statistical analysis. For primary samples, Wilcoxon score test and chi-square test were used. Mann-Whitney $U$ test was performed for assessing statistical differences in parental and chemo-resistant cell lines.

Clinical end-points were response rate, PFS and overall survival (OS). Tumour assessment by computed tomography scan was performed every 2-3 months until progressive disease. Patients without a second computed tomography evaluation were not assessable for response rate. PFS was defined as the time of the initial first-line chemotherapy dose until disease progression, death from any cause or administrative end of follow-up, whichever occurs first. Overall survival was defined as the time from first-line chemotherapy initiation to death for any cause or administrative end of follow-up, whichever is first. Kaplan-Meier curves were used to plot PFS and OS. Cox proportional hazard regression was used to perform the survival analysis. All $P$-values were two-sided. Analysis was performed by using SAS V9.4 (SAS Institute, Cary, NC, USA) statistical software.

\section{RESULTS}

Nuclear expression of $p$-IGF-1R predicts poor survival in $\mathrm{mCRC}$ patients treated with first-line chemotherapy and targeted agents. First, we evaluated the pattern of p-IGF-1R expression by IHC in 563 samples from mCRC patients. Representative cases of membrane, perinuclear, negative and exclusive nuclear p-IGF1R IHC staining are shown in Figure 1A. Nuclear p-IGF-1R staining was present in $35 \%$ of patients in the SIEMENS-2 cohort, in $36 \%$ in the BECOX trial and in $16 \%$ of patients in the PULSE and POSIBA trials. The frequency of nuclear p-IGF-1R expression was significantly higher in $B R A F$ mutant (49\%) compared to RAS mutant (14\%) or double wild-type (2WT) (23\%) patients $(P<0.0001)$. In addition, cases with p-IGF-1R expression identified exclusively in the nucleus were higher in BRAF mutant (16\%) than in RAS mutant $(2 \%)$ or $2 \mathrm{WT}(4 \%)$ patients $(P<0.0001)$ (Supplementary Table S1). A summary of the patient characteristics is shown in Supplementary Table S1. For those patients with available clinical information $(n=470)$, our analysis showed that nuclear p-IGF-1R was associated with poor eastern cooperative oncology group scale of performance status $(P=0.001)$, high alkaline phosphatase levels $(P=0.013)$ and $B R A F$ mutation $(P<0.0001)$ (Table 1$)$.

For 44 mCRC patients, primary tumour and paired metastatic biopsies (second or third biopsies) were available. Paired biopsies 
were obtained from patients without previous therapy for advanced disease $(n=12)$, after treatment with chemotherapy alone $(n=27)$, or after treatment with chemotherapy and anti-EGFR $(n=5)$. The concordance rate of $\mathrm{p}$-IGF-1R in the nucleus assessed by IHC analysis between primary and paired metastases was $98 \%$ (Figure 1B). An increment of the nuclear p-IGF-1R in the metastasis compared to its matched primary samples was observed in $11 \%$ of the cases. Representative images of the increased levels of nuclear p-IGF-1R expression in metastatic samples (after chemotherapy and after chemotherapy and antiEGFR therapy) compared to the matched primary untreated tumours are shown in Figure 1C. Of note, none of the initially nuclear p-IGF-1R negative patients in the primary tumour became positive at its paired metastases, regardless of which treatment they received.

Mutational status for BRAF and KRAS was also assessed. Fortysix patients $(10 \%)$ that presented the V600E mutation in $B R A F$, showed a significantly inferior OS (median 11.2 months [95\% CI, 7.6-14.9]) compared to $2 \mathrm{WT}$ disease (median 30.7 months [95\% CI 26.1-35.2]) and RAS mutant disease (median 25 months [95\% CI 14.9-35.1]) $(P<0.0001)$. Kaplan-Meier analysis estimated that there were no differences in PFS according to the p-IGF-1R nuclear expression (Figure 2A). However, the presence of high expression nuclear p-IGF-1R $(\geqslant 20 \%)$ showed a significantly inferior OS (median 16.7 months [95\% CI, 13.4-20.2]) compared to low (1-19\%) (median 24.6 months [95\% CI 17.8-31.5]) and negative p-IGF-1R nuclear expression (median 30.5 months [95\% CI $26.3-$ 31.7]) $(P<0.01)$ (Figure 2B). Nevertheless, there was a significantly poorer PFS according to BRAF mutation (Figure $2 \mathrm{C}$ ). The presence of BRAF mutation (median 11.2 months [95\% CI 6.9-15.5]) or high expression of nuclear p-IGF-1R $(\geqslant 20 \%)$ in wild-type $B R A F$ patients (median 17.5 months [95\%CI 12.9-22.1]) showed a significantly inferior OS compared to $B R A F$ wild-type patients with low p-IGF-1R (1-19\%) (median 27.2 months [95\% CI 15.1-39.3]) and negative $\mathrm{p}-\mathrm{IGF}-1 \mathrm{R}$ nuclear expression (median 31.3 months [95\% CI 27.1-35.6]) $\quad(P<0.0001) \quad$ (Figure 2D). In the Cox proportional hazards model, nuclear p-IGF-1R was not significant for PFS (Supplementary Table S2) nor OS (Table 2).

Chronic oxaliplatin treatment leads to acquired drug resistance and induces IGF-1R nuclear localisation. In order to further characterise how nuclear localisation of IFG-1R was associated with treatment resistance, Colo320, DLD-1, HCT116, HT29, SW1116, SW480, SW403, SW1463, and SW837 CRC cell lines were treated with increasing concentrations of oxaliplatin and 5FU at physiologically administrated concentrations. Dose-response
A
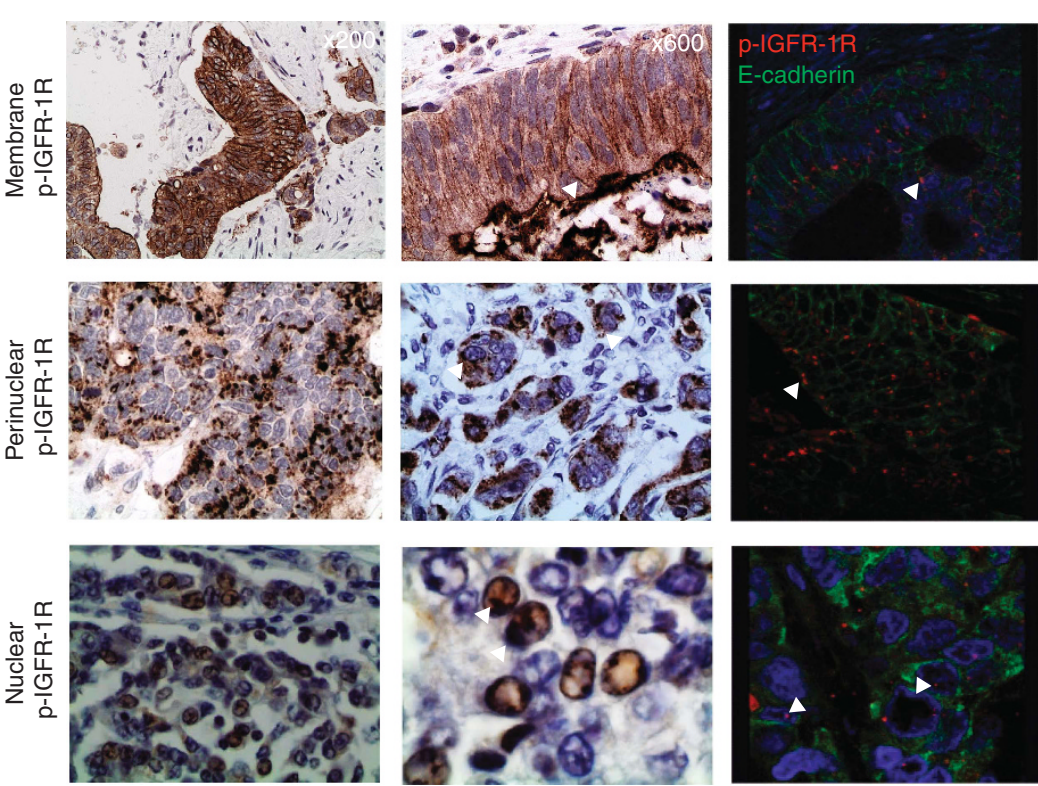
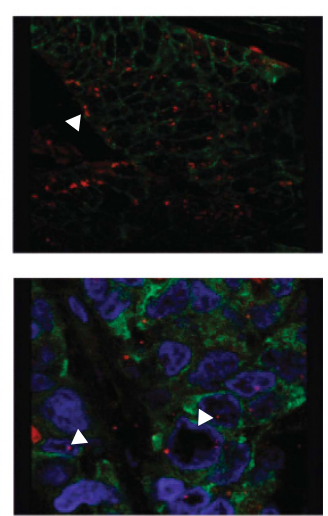

C
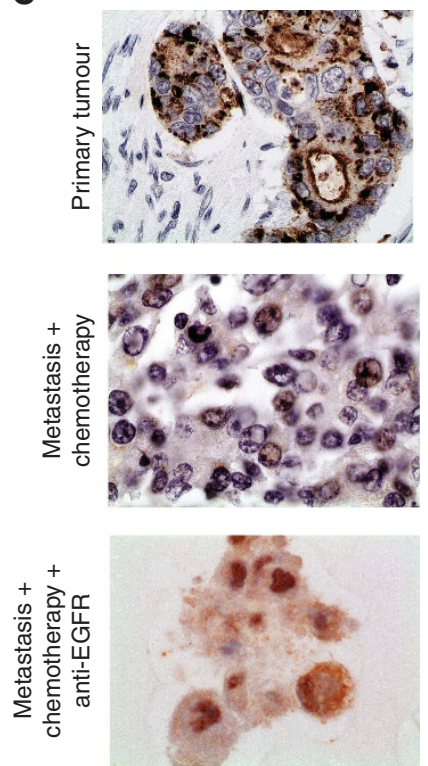

B

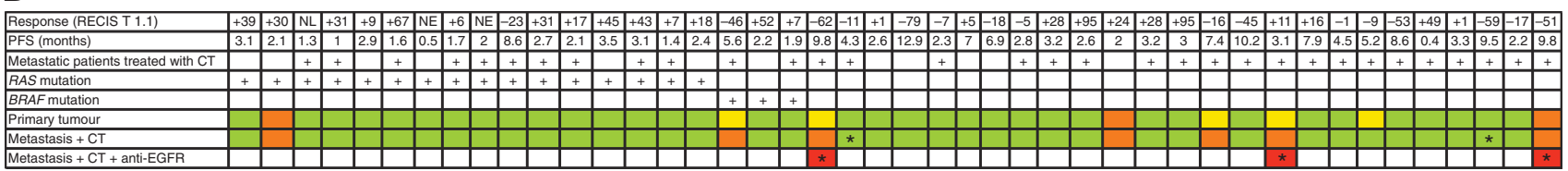

Figure 1. Expression patterns of IGF-1R: nuclear expression of p-IGF-1R increases after chemotherapy exposure but only in patients with initial p-IGF-1R nuclear expression. (A) Patterns of expression of p-IGF-1R analysed with optical and confocal immunofluorescence microscopy. Arrowheads denote foci of membrane p-IGF-1R expression (top panel), granular-perinuclear and dot-like endoplasmic reticulum and Golgi patterns (middle panel), and nuclear p-IGF-1R staining (lower panel) observed in some tumours. Confocal microscope immunofluorescence shows p-IGF-1R in red, E-cadherin (green), and nuclei stained in TO-PRO-3. (B) Summary nuclear p-IGF-1R expression. RAS and BRAF mutational status in 44 pairs of primary and metastatic samples of colorectal carcinoma is also shown. Clinical data are summarised in top rows, including response rate by RECIST 1.1 criteria, PFS, and collection of the biopsy after treatment chemotherapy or chemotherapy plus anti-EGFR. The bottom three rows indicate the presence of nuclear p-IGF-1R. In green are indicated negative samples ( $0 \%$ of cells positive), in yellow $(<20 \%)$, orange (20-50\%), and red $(>50 \%)$ are indicated samples with different percentage measured by IHC. Asterisks indicated biopsies obtained after anti-EGFR therapy. (C) Representative sections from IHC analysis of nuclear p-IGF-1R. Top row shows the distribution of p-IGF-1R in the primary tumour collected before treatment; a metastatic biopsy collected after chemotherapy (oxaliplatin and 5-fluorouracil) treatment is indicated in the middle row, and a representative metastatic biopsy collected after chemotherapy (including oxaliplatin, 5-fluorouracil, irinotecan) and anti-EGFR (cetuximab or panitumumab) treatment is in the bottom of the panel. 
Table 1. Patient characteristics according to nuclear p-IGF-1R expression

\begin{tabular}{|c|c|c|c|c|}
\hline Characteristics & $\begin{array}{l}\text { Nuclear } \\
\text { p-IGF-1R } \\
\text { negative }\end{array}$ & $\begin{array}{l}\text { Nuclear } \\
\text { p-IGF-1R } \\
\text { low }\end{array}$ & $\begin{array}{l}\text { Nuclear } \\
\text { p-IGF-1R } \\
\text { high }\end{array}$ & $P$-value \\
\hline Number; $n=470$ & 360 & 68 & 42 & \\
\hline Gender (female) & 121 & 26 & 15 & 0.75 \\
\hline Age, median (s.d.) & $63.3(10.4)$ & $61.4(10.1)$ & $60.1(10.6)$ & 0.88 \\
\hline $\begin{array}{l}\text { Surgery of primary } \\
\text { (yes) }\end{array}$ & 244 & 54 & 26 & 0.09 \\
\hline $\begin{array}{l}\text { Primary location } \\
\text { (right side) }\end{array}$ & 79 & 16 & 13 & 0.405 \\
\hline $\begin{array}{l}\text { ECOG PS } \\
0 \\
1 \\
2\end{array}$ & $\begin{array}{r}202 \\
143 \\
14\end{array}$ & $\begin{array}{r}30 \\
35 \\
3\end{array}$ & $\begin{array}{r}14 \\
21 \\
7\end{array}$ & 0.001 \\
\hline $\begin{array}{l}\text { Leucocyte count } \\
>10000\end{array}$ & 73 & 18 & 8 & 0.45 \\
\hline $\begin{array}{l}\text { Alkaline phosphatase } \\
>300 \mathrm{UI}^{-1}\end{array}$ & 58 & 20 & 12 & 0.013 \\
\hline $\begin{array}{l}\text { Lactate } \\
\text { dehydrogenase } \\
>\text { ULN }\end{array}$ & 107 & 20 & 16 & 0.13 \\
\hline CEA, median & 334.9 & 705.1 & 316.9 & 0.84 \\
\hline Metastases, sites $>1$ & 176 & 36 & 21 & 0.94 \\
\hline Liver M1 & 290 & 54 & 31 & 0.38 \\
\hline Peritoneal M1 & 66 & 12 & 14 & 0.062 \\
\hline $\begin{array}{l}\text { Genotype } \\
\text { BRAF } \\
\text { RAS } \\
2 W T\end{array}$ & $\begin{array}{r}20 \\
90 \\
250\end{array}$ & $\begin{array}{r}8 \\
17 \\
43\end{array}$ & $\begin{array}{r}10 \\
2 \\
30\end{array}$ & $<0.0001$ \\
\hline $\begin{array}{l}\text { GEMCAD stage } \\
\text { Low-risk } \\
\text { Intermediate-risk } \\
\text { High-risk }\end{array}$ & $\begin{array}{r}76 \\
183 \\
67 \\
\end{array}$ & $\begin{array}{r}15 \\
35 \\
8 \\
\end{array}$ & $\begin{array}{r}9 \\
20 \\
13 \\
\end{array}$ & 0.081 \\
\hline \multicolumn{5}{|c|}{$\begin{array}{l}\text { Abbreviations: CEA = carcinoembryonic antigen; ECOG PS = Eastern Cooperative Oncol- } \\
\text { ogy Group Scale of Performance Status; GEMCAD = Grupo Español Multidisciplinar en } \\
\text { Cáncer Digestivo; IGF-1R=insulin-like growth factor-1 receptor; ULN=upper limit of } \\
\text { normal. }\end{array}$} \\
\hline
\end{tabular}

plots showed different degrees of sensitivity to the drugs (Supplementary Figure S1A). In addition, higher concentrations of these drugs were tested in DLD-1, HT29, and their chemoresistant derivates, DLD-1-OxR and HT29-OxR, showing that the latest exhibit specific resistance to oxaliplatin alone (Supplementary Figure S1B and C). Next, we wanted to assess whether the localisation of p-IGF-1R in the set of cell lines validated the results observed in the primary samples. By IHC of formalin-fixed paraffin-embedded cellular blocks, we showed an association between resistance to oxaliplatin and 5-FU and the amount of p-IGF-1R in the nuclear compartment $(R=0.604$ and 0.738 for the nine CRC cell lines and for the set of DLD-1, DLD-1-OxR, HT29, HT29-OxR, SW1116 and HCT116, respectively (Figure 3A and Supplementary Figures S1A and B)). To further confirm this finding, we performed confocal microscopy immunofluorescence with antibodies against IGF-1R and p-IGF-1R. These results corroborated the increase of p-IGF-1R in the nucleus of HT29OxR compared to the parental HT29 cell line (Figure 3B). This enrichment of the nuclear IGF-1R in the chemo-resistant HT29OxR and SW1116 in comparison to the chemo-sensitive HT29, HCT116, and Colo320 was also slightly observed by western blot (Supplementary Figures S1D and E).

To determine whether oxaliplatin had any effects on downstream targets of the IGF-1R signalling pathway, activation of AKT and IRS-1 were evaluated. In a time-response experiment using HT29 and HT29-OxR cell lines, p-AKT and p-IRS-1 are inhibited $5 \mathrm{~min}$ after the oxaliplatin treatment $(20 \mu \mathrm{M})$ was initialised, and this inhibition was sustained up to $60 \mathrm{~min}$ in the parental HT29; however, no effect was seen in the HT29-OxR (Supplementary Figure S1F). In addition, we showed that low dosage of oxaliplatin after 1 -h exposure results in a reduction of p-AKT in HT29, but not in HT29-OxR cells (Supplementary Figure S1G).

Targeted therapies increase the localisation of IGF-1R within the nuclear compartment. As clinical data suggested a correlation between resistance to anti-EGFR compounds and nuclear internalisation of IGF-1R, we hypothesised that these could also be the cause of resistance to other targeted therapies, such as ganitumab (anti-IGF-1R antibody), sorafenib (tyrosine kinase inhibitor of VEGFR and PDGFR), and the combination of both. Despite cellular viability was compromised upon exposure to both drugs compared to untreated cells, no significant differences were observed between sensitive and resistant cell lines (Supplementary Figure S2A). Nevertheless, when we assessed the signalling pathway activity, our results showed that ganitumab, but not the metalloproteinase inhibitor TIMP1 or sorafenib, inhibited the activation of AKT and IRS-1, thus suggesting a compromise of the IGF-1R intracellular molecular pathway upon treatment with an anti-IGF-1R antibody (Figure 3C). In addition, depletion of the IGF-1R signalling pathway activated apoptosis in the parental HT29 cell line; however, in the chemo-resistant HT29-OxR line, apoptosis was not activated (Supplementary Figure 2B).

Subsequently, we aimed at assessing whether exposure to ganitumab, the anti-IGF-1R monoclonal antibody, and the compound NVP-AEW541, a tyrosine kinase inhibitor of IGF-1R, might play a role in the cellular localisation. Our results showed that ganitumab, but not NVP-AEW541, decreased the expression of IGF-1R in the cytosolic fraction. However, despite the fact that expression of IGF-1R in the nuclear compartment was slightly higher in HT29-OxR compared to the parental HT29 cells, such levels of nuclear IGF-1R were significantly increased upon treatment with ganitumab in both HT29 and HT29-OxR cell lines (Figure 3D). By immunofluorescence and confocal microscopy analysis, we did validate that total cytosolic IGF-1R expression was depleted upon exposure to ganitumab; however, levels of p-IGF-1R showed an increase in the nuclear compartment of HT29-OxR cells treated with ganitumab but not NVP-AEW541 (Figure 3E). These results were further confirmed using the parental DLD-1 and its oxaliplatin-resistant induced cell line (Supplementary Figure S2C). Furthermore, cetuximab increased the presence of nuclear IGF-1R in HT29-OxR cell line but did not in the parental HT29 cell line (Supplementary Figure S2D). Interestingly, the addition of dasatinib and dynasore, a SRC inhibitor and an inhibitor of endocytic vesicle formation, respectively, dramatically increased IGF-1R in the nuclear compartment of the HT29-OxR cell line, while only a slight increase was observed in the parental HT29 cells (Figure 3F). Finally, when these two compounds were combined with ganitumab, the presence of nuclear IGF-1R in HT29-OxR cells became even more prominent, indicating that exposure to dasatinib and dynasore might not be sufficient to impede the nuclear localisation of IFG-1R after treatment with ganitumab.

Loss-of-function of PIAS3 and treatment with curcumin and leptomycin inhibits IGF-1R nuclear sequestration. In order to explore the potential molecular mechanism involved in the translocation of IGF-1R into the nucleus when cells are exposed to chemo-therapeutic compounds and therefore potentially inducing acquired resistance, we sought to investigate the levels of the protein inhibitor of activated STAT3 (PIAS3), as this protein is known to retain the IGF-1R inside the nuclear compartment 
A

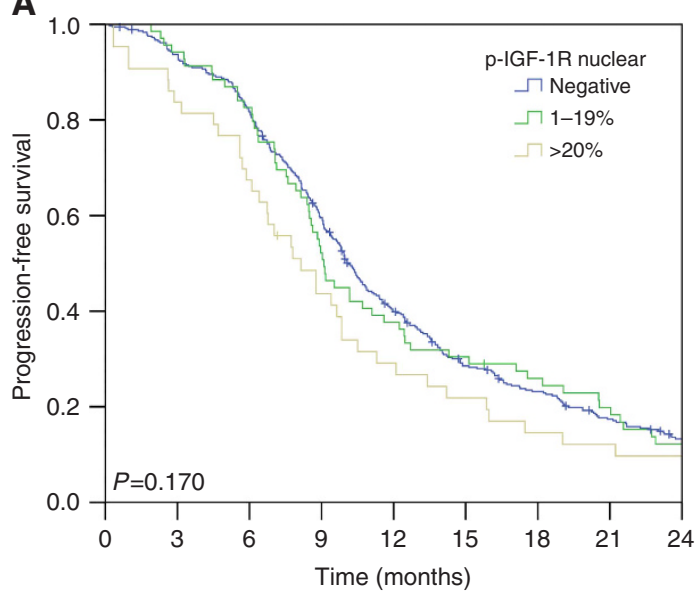

C

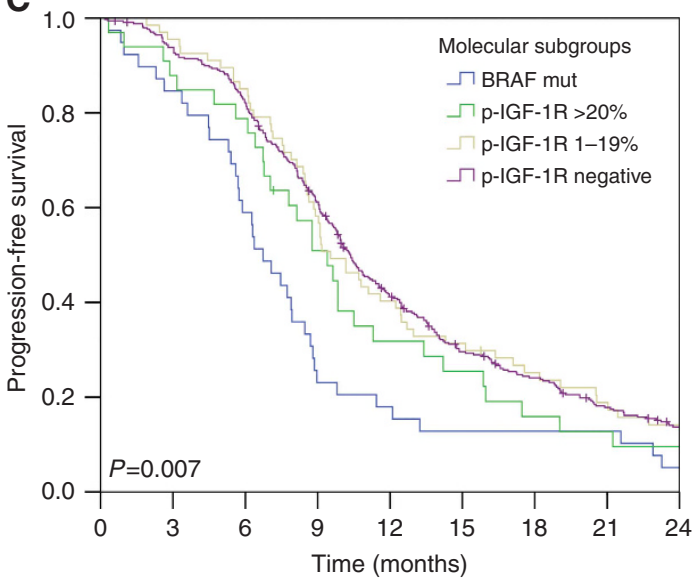

B

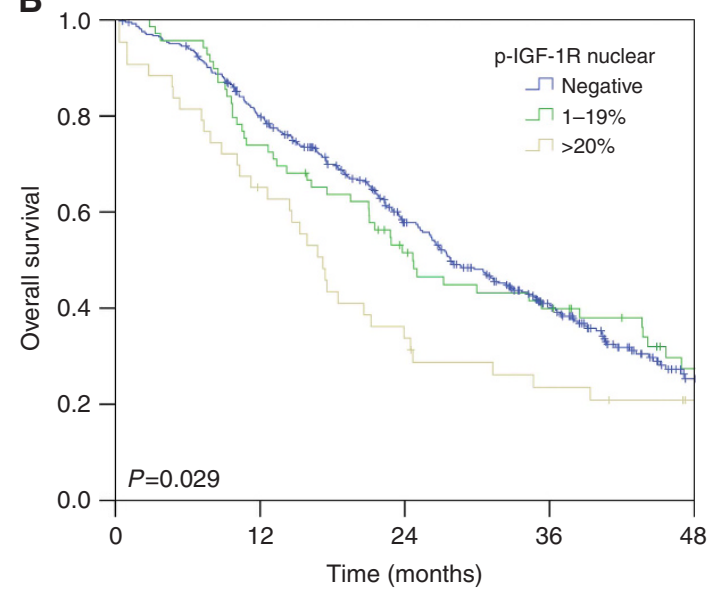

D

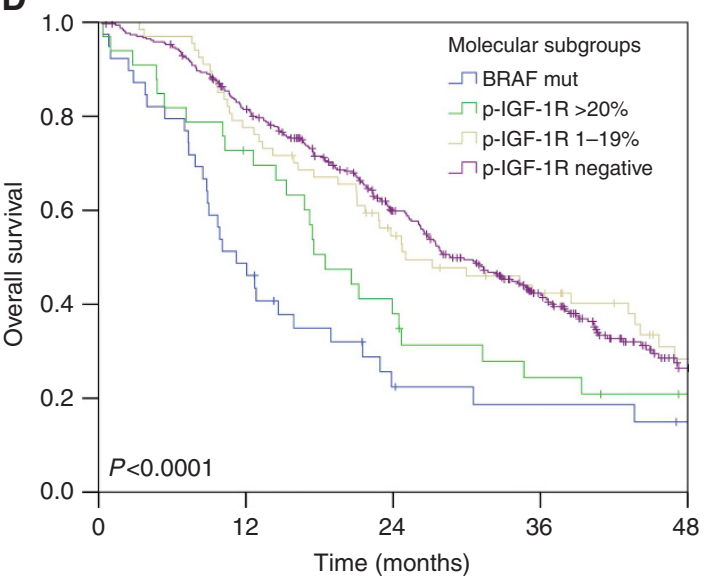

Figure 2. Prognostic role of nuclear p-IGF-R in mCRC. PFS and OS according to nuclear p-IGF-1R location. In blue-0, patients with negative p-IGF$1 \mathrm{R}$ nuclear expression; in red-1, patients with low positivity ( $<20 \%)$ p-IGF-1R nuclear expression; in green-2, patients with high positivity ( $\geqslant 20 \%)$ p-IGF-1R nuclear expression. (A) Kaplan-Meier curves of PFS according to (negative vs $\leqslant 20 \%$ vs $>20 \%$ nuclear p-IGF-1R). (B) Kaplan-Meier curves of OS for patients with tumours (negative vs $\leqslant 20 \%$ vs $>20 \%$ nuclear p-IGF-1R). (C) Kaplan-Meier curves of PFS according to BRAF and non-BRAF mutant CRC (negative vs $\leqslant 20 \%$ vs $>20 \%$ nuclear $p-I G F-1 R$ ). (D) Kaplan-Meier curves of OS according to BRAF and non-BRAF mutant CRC (negative vs $\leqslant 20 \%$ vs $>20 \%$ nuclear $p-I G F-1 R$ ).

(Sundvall et al, 2012). Our results revealed that nuclear PIAS3 expression increased after treatment with ganitumab, NVP-AEW541 and both compounds in HT29-OxR-resistant cells, thus supporting the hypothesis that PIAS3 might be involved in the IGFR-1R internalisation (Figure 4A).

In order to block the nuclear retention of IGF-1R, RNAi experiments were performed. In fact, siRNAs against PIAS3 were assayed in HT29-OxR and DLD-1-OxR cell lines demonstrating loss-of-function at the protein level after $72 \mathrm{~h}$ post-transfection (Figure 4B). Our results showed that the presence of nuclear IGF$1 \mathrm{R}$ was significantly suppressed upon silencing of PIAS3 (Figure 4B and C). We then exposed HT29 and HT29-OxR cells to curcumin for $24 \mathrm{~h}$. Curcumin has been suggested to interact with PIAS3 in order to boost the negative effect on STAT3 activity (Saydmohammed et al, 2010). Our results showed that curcumin avoids, at least partially, nuclear localisation of IGF-1R in both cell lines (Figure 4D). Similarly, when cells were treated with leptomycin B, a specific nuclear traffic inhibitor through CRM1, and leptomycin B with ganitumab for $24 \mathrm{~h}$, we observed a decrease in the levels of nuclear IGF-1R in HT29 and HT29-OxR (Figure 4E). Altogether, our data indicated that PIAS3 might be playing an active role in the maintenance of IGF-1R inside the nucleus, and this phenomenon could potentially explain acquired resistance.

\section{DISCUSSION}

A subset of CRC patients without BRAF mutations have a gene expression signature and a prognosis similar to those presenting $B R A F$ mutations (aka., BRAF-like). In addition, roughly $13 \%$ of $2 \mathrm{WT}$ and $30 \%$ of KRAS mutant localised CRC patients also show a $B R A F$-like signature (Popovici et al, 2012). Despite the fact that the $B R A F$ mutation is a solid poor prognostic marker, BRAF-like tumours might show deregulation of specific genes such as RANBP2 (Vecchione et al, 2016), RAC1b (Alonso-Espinaco et al, 2014), genes belonging to the CMS4 (Barras et al, 2017) or nuclear internalisation of IGF-1R, which could also contribute to the poor prognosis. In the present study, nuclear IGF-1R did not reach significance in the multivariate analysis; however, we have found that mCRC patients with BRAF mutations are significantly enriched for the expression of nuclear IGF-1R. Because it has been recently shown that RANBP2 is essential for survival in $B R A F$-like phenotype and is implicated also in IGF-1R internalisation (Vecchione et al, 2016), we hypothesise that nuclear IGF-1R might constitute a marker to easily identify BRAF-like phenotype in $\mathrm{mCRC}$.

Furthermore, we have investigated the patterns of IGF-1R expression and whether IGF-1R localisation could be involved in 
Table 2. OS, multivariate analysis for nuclear p-IGF-1R

\begin{tabular}{|c|c|c|c|c|}
\hline & \multicolumn{2}{|c|}{ Model $1^{a}$} & \multicolumn{2}{|l|}{ Model $2^{b}$} \\
\hline & HR & $P$-value & HR & $P$-value \\
\hline \multicolumn{5}{|c|}{ Nuclear p-IGF-1R } \\
\hline Negative & Ref. & & Ref. & \\
\hline Low & $1.12(0.81-1.54)$ & 0.49 & $1.17(0.85-1.60)$ & 0.33 \\
\hline High & $1.33(0.91-1.94)$ & 0.14 & $1.38(0.95-2.01)$ & 0.09 \\
\hline \multicolumn{5}{|c|}{ Gender (female) } \\
\hline Age $>65$ & & & $1.38(1.08-1.77)$ & 0.011 \\
\hline $\begin{array}{l}\text { Surgery of } \\
\text { primary (yes) }\end{array}$ & $0.64(0.48-0.85)$ & 0.0021 & $0.67(0.51-0.89)$ & 0.0054 \\
\hline $\begin{array}{l}\text { Primary location } \\
\text { (right side) }\end{array}$ & $1.35(1.01-1.79)$ & 0.041 & & \\
\hline \multicolumn{5}{|l|}{ ECOG PS } \\
\hline 0 & Ref. & & Ref. & \\
\hline 1 & $2.36(1.81-3.10)$ & $<0.0001$ & $2.42(1.87-3.13)$ & $<0.0001$ \\
\hline 2 & $8.49(4.69-15.37)$ & $<0.0001$ & $8.38(4.69-14.97)$ & $<0.0001$ \\
\hline $\begin{array}{l}\text { Leucocyte count } \\
>10000\end{array}$ & $1.44(1.08-1.92)$ & 0.013 & $1.54(1.16-2.04)$ & 0.0027 \\
\hline $\begin{array}{l}\text { Alkaline } \\
\text { phosphatase } \\
>300 \mathrm{UI}^{-1}\end{array}$ & $1.26(0.88-1.80)$ & 0.21 & & \\
\hline $\begin{array}{l}\text { Lactate } \\
\text { dehydrogenase } \\
>\text { ULN }\end{array}$ & $1.09(0.74-1.60)$ & 0.65 & & \\
\hline $\begin{array}{l}\text { Metastases, sites } \\
>1\end{array}$ & $0.94(0.71-1.26)$ & 0.69 & & \\
\hline \multicolumn{5}{|l|}{ Liver M1 } \\
\hline Peritoneal M1 & $1.34(0.98-1.83)$ & 0.067 & $1.46(1.10-1.95)$ & 0.0098 \\
\hline \multicolumn{5}{|l|}{ Genotype } \\
\hline RAS & $1.31(0.96-1.79)$ & 0.086 & $1.31(0.97-1.76)$ & 0.081 \\
\hline $\begin{array}{l}\text { BRAF } \\
2 W T\end{array}$ & $\begin{array}{c}2.29(1.52-3.44) \\
\text { Ref. }\end{array}$ & $<0.0001$ & $\begin{array}{c}2.66 \text { (1.77-3.99) } \\
\text { Ref. }\end{array}$ & $<0.0001$ \\
\hline \multicolumn{5}{|c|}{ GEMCAD stage } \\
\hline $\begin{array}{l}\text { Low-risk } \\
\text { Intermediate- } \\
\text { risk }\end{array}$ & $\begin{array}{c}\text { Ref. } \\
2.10(1.44-3.07)\end{array}$ & 0.0001 & $\begin{array}{c}\text { Ref. } \\
1.84(1.31-2.59)\end{array}$ & 0.0004 \\
\hline High-risk & $1.54(0.90-2.65)$ & 0.12 & $1.73(1.11-2.71)$ & 0.016 \\
\hline \multicolumn{5}{|c|}{$\begin{array}{l}\text { Abbreviations: ECOG PS = Eastern Cooperative Oncology Group Scale of Performance } \\
\text { Status; GEMCAD = Grupo Español Multidisciplinar en Cáncer Digestivo; } H R=\text { hazard ratio; } \\
\text { IGF-1R=insulin-like growth factor- } 1 \text { receptor; OS = overall survival; ULN = upper limit of } \\
\text { normal. } \\
\text { a Model } 1 \text { includes those variables with a P-value }<0.05 \text { in the univariate analysis. The } \\
\text { variable nuclear } p-I G F-1 R \text { is forced into the model. } \\
b_{M} \text { Model } 2 \text { selects variables using a stepwise approach with a } P \text {-value of } 0.2 \text { to enter into the } \\
\text { model and of a multivariate } P \text {-value }<0.05 \text { to stay in the model. The variable nuclear p-IGF- } \\
1 R \text { is forced into the model. }\end{array}$} \\
\hline
\end{tabular}

mechanisms of chemotherapeutic resistance in mCRC. Assessing the distribution of IGF-1R in a panel of 11 cell lines and $44 \mathrm{mCRC}$ samples, we observed that chemotherapy-resistant cells and pretreated mCRC paired biopsies showed an increment in the nuclear expression of IGF-1R compared to sensitive cell lines and untreated patients. We inhibited IGF-1R nuclear expression with the monoclonal antibody IGF-1R, ganitumab, and the IGF-1R tyrosine kinase inhibitor, NVP-AEW541. Despite the fact that ganitumab induces cytotoxicity and inhibits phosphorylation of IRS-1 and AKT in both HT29 and HT29-OxR, the oxaliplatinresistant line did not undergo apoptosis. Previously published data showed that IGF-1R kinase activity was required for IGF-1R to enter the nucleus (Aleksic et al, 2010; Sehat et al, 2010). Consequently, these authors observed that tyrosine-kinase IGF$1 \mathrm{R}$ inhibition suppressed IGF-1R internalisation. Interestingly, our results showed that nuclear IGF-1R increased after ganitumab but not with NVP-AEW541 treatment in HT29-OxR and DLD-1-OxR cell lines. In addition, we have also observed that other compounds shown to inhibit EGFR nuclear internalisation such as the SRC inhibitor (dasatinib) (Li et al, 2009) also increased IGF-1R nuclear expression.

Previous data implicated sumoylation proteins such as the SUMO ligases (Ubc9 and RANBP2) in IGF-1R internalisation (Sehat et al, 2010; Packham et al, 2014). We show here for the first time that PIAS3 (another critical protein in SUMO E3 process) is also implicated in IGF-1R nuclear internalisation in colorectal cancer chemotherapy-resistant cells. We observed that PIAS3 increases after treatment with ganitumab and NVP-AEW541, and this increment is still more noticeable in oxaliplatin refractory cell lines. It has been previously published that PIAS3 promotes Sumoylation and nuclear sequestration of ErbB4 protein (Sundvall et al, 2012). Accordingly, after silencing PIAS3, we observed a significant reduction of IGF-1R in the nucleus. In addition, we have shown that curcumin and leptomycin $\mathrm{B}$ also reduce the presence of IGF-1R in the nucleus, which is in agreement with the previously reported effect of this compound on RANBP1 (Niu et al, 2013). The consequences of IGF-1R nuclear translocation in cancer cells are still unclear. As it has been recently described, IGF$1 \mathrm{R}$ in the nucleus could be involved in the transcription regulation of other genes (Sehat et al, 2010), and increase the promoter activity of LEF1 downstream target genes Cyclin D1 and Axin 2 in in vitro models of melanoma, cervical cancer and non-small cell lung carcinoma (Aleksic et al, 2010). This phenomenon might be of the utmost importance in colorectal cancer cells as per their dependence on the WNT signalling pathway.

To our knowledge, these results represent the first clinical and functional description of how nuclear IGF-1R expression might play an active role in the acquired resistance to conventional chemotherapy (FOLFOX, CAPOX and FOLFIRI) and targeted therapies in mCRC. We hypothesise that patients with nuclear IGF-1R (representing only $14 \%$ of RAS mutant and $23 \%$ of $2 \mathrm{WT}$, but $49 \%$ of $B R A F$ mutant $\mathrm{mCRC}$ ) are characterised by constitutional overexpression of $R A N B P 2$, which facilitates the presence of IGF-1R in the nucleus. After chemotherapy and targeted therapies (ganitumab, NVP-AEW541 and dasatinib), PIAS3 is increased in the nuclear compartment and contributes to the increased expression of IGF-1R in the nucleus, which results in the activation of the WNT pathway, previously related to chemoradiotherapy resistance (Supplementary Figure S3) (Kendziorra et al, 2011).

In conclusion, we demonstrate that IGF-1R nuclear translocation is associated with resistance to chemotherapy and targeted therapies both in vitro and clinically in mCRC patients, that nuclear translocation is a process depending on PIAS3, and also that nuclear IGF-1R might have the potential to be used as a biomarker for poor prognosis.

\section{ACKNOWLEDGEMENTS}

We thank to Drs Cristobal Mezquita, Antonio Postigo and Mayumi Ono for the scientific revision of the manuscript. We also thank Dr Albert Abad from Hospital Germans Trias i Pujol (Barcelona, Spain) for kindly providing cell lines and reagents. This work was supported by Instituto de Salud Carlos III and co-funded by the European Regional Development Fund (ERDF) (PI13/01728 to JM, CP13/00160 and PI14/00783 to JC), Mutua Madrileña (AP75002010 to JM), the European Comission (COLONGEVA to JC), Asociación Española Contra el Cáncer (to JC), the Agència de Gestió d'Ajuts Universitaris i de Recerca (2014 SGR 0903 and 2014 SGR 135) and CERCA Programme/Generalitat de Catalunya, and CIBERehd. Technical support was provided by the Banc de Tumors-Biobanc Hospital Clinic-IDIBAPS and Xarxa de Bancs de Tumors de Catalunya (XBTC) sponsored by Pla Director d'Oncologia de Catalunya. 
A

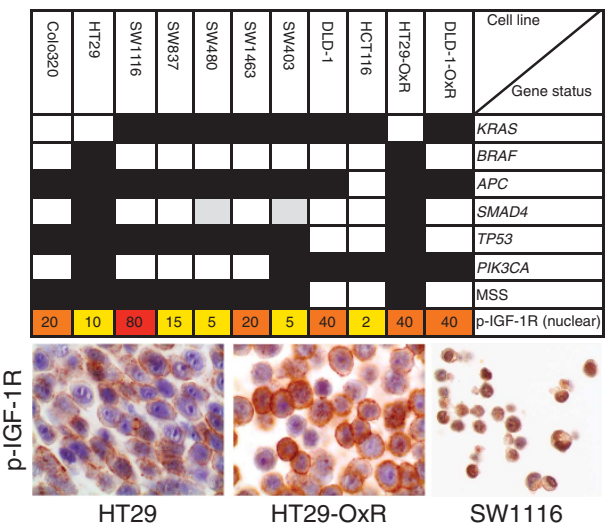

C

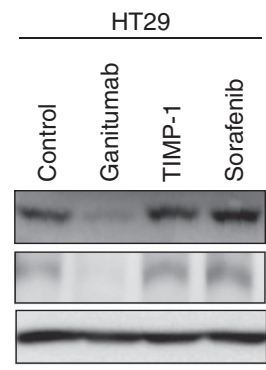

E

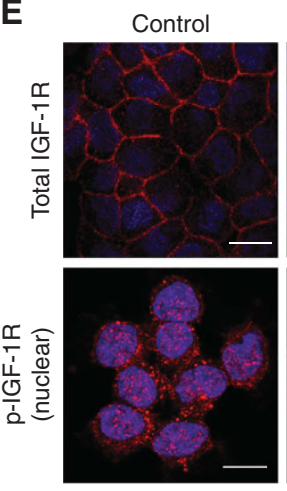

HT29-OxR
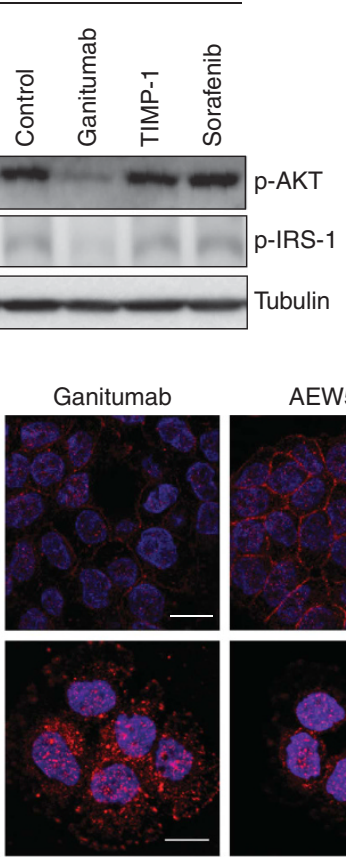

B
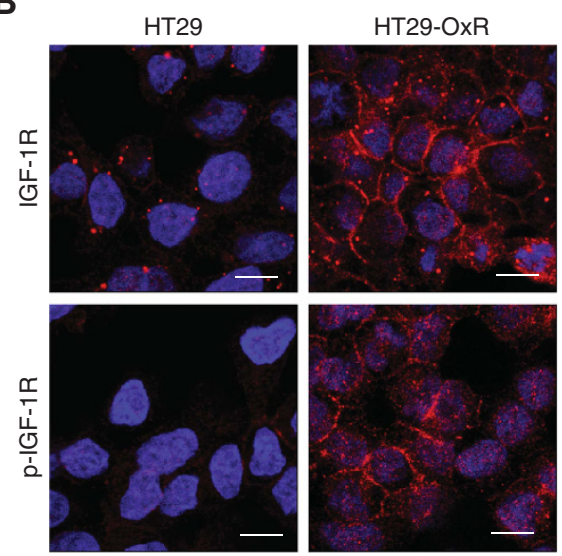

D
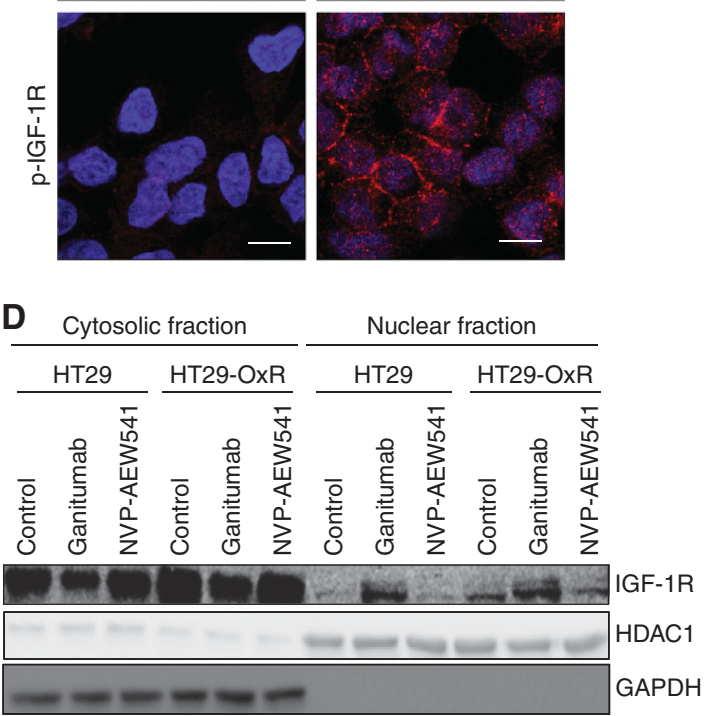

$\mathbf{F}$
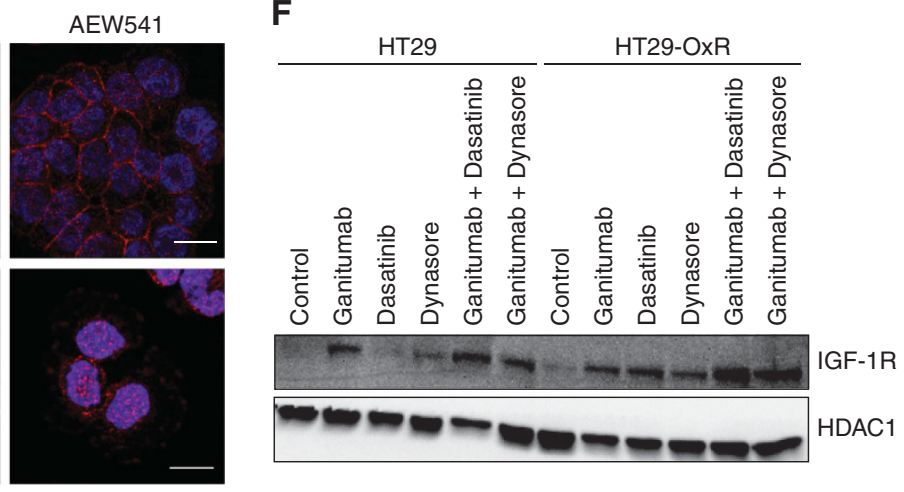

Figure 3. Implications of targeted therapeutic agents in IGF-1R expression. (A) Summary of the mutational status of several genes implicated in the colorectal carcinogenesis in the panel of CRC cell lines used in this study. Black indicates the presence of mutation, grey indicates deletion, and white indicates wild-type. In yellow $(<20 \%)$, orange $(20-50 \%)$ and red ( $>50 \%)$ is shown the gradient of nuclear p-IGF-1R measured by immunohistochemistry. Numbers indicate the \% of nuclear p-IGF-1R positivity. Representative images of HT29, HT29-OxR, and SW1116 illustrate differences in the staining distribution and localisation of p-IGF-1R. (B) Confocal microscopy indicative immunofluorescence images of the location of total IGF-1R and phospho-IGF-1R in HT29 and HT29-OxR cell lines. Fixed cells were stained with anti-IGF-1R or anti-p-IGF-1R antibodies (red) and DAPI (blue) for nuclear counterstaining. Graphs show the percentage of viable cells $72 \mathrm{~h}$ after incubation with increasing dosages of a combination of 5-FU and oxaliplatin in several CRC cell lines. Each data point represents the average value from three independent experiments. (C) Total cells lysates from HT29 and HT29-OxR cell lines were immunoblotted against anti-p-AKT and anti-p-IRS-1 to evaluate the effect of a 24-h exposure to ganitumab $(5 \mu \mathrm{M})$, TIMP1 $(100 \mu \mathrm{M})$, and sorafenib $(2 \mu \mathrm{M})$ on the IGF-1R signalling pathway. Tubulin was used as loading control. (D) Immunoblot analysis of IGF-1R levels of nuclear and cytosolic protein extracts from HT29 and HT29-OxR cell lines were evaluated after incubation for $24 \mathrm{~h}$ with ganitumab $(5 \mu \mathrm{M})$ and NVP-AEW541 $(0.1 \mu \mathrm{M})$. Antibodies anti-HDAC1 and anti-GAPDH were used as controls for nuclear and cytosolic fractions, respectively. Note the increase of IGF-1R in the nuclear compartment after treatment with the monoclonal antibody against IGF-1R. (E) Representative confocal microscopy images of total and phospho-IGF-1R expression in HT29-OxR cells after incubation with ganitumab $(5 \mu \mathrm{M})$ or NVP-AEW541 $(0.1 \mu \mathrm{M})$ for $24 \mathrm{~h}$. Cells were immunostained with IGF-1R or p-IGF-1R antibodies (red) and DAPI (blue) for nuclear counterstaining. While membrane located IGF-1R was inhibited after treatment with ganitumab, nuclear IGF-1R markedly increased in HT29-OxR cells. (F) Immunoblot analysis of nuclear IGF-1R in HT29 and HT29-OxR cell lines after incubation with ganitumab (5 $\mu \mathrm{m})$, dasatinib (50 nM), dynasore ( $30 \mathrm{~nm}$ ) and their combination for $24 \mathrm{~h}$. Note that in HT29-OxR, but not HT29 cells, the presence of dasatinib and dynasore without ganitumab was able to induce expression of IGF-1R expression in the nucleus. 
A

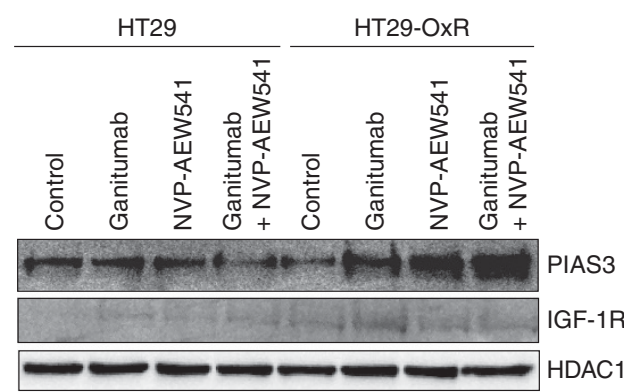

B

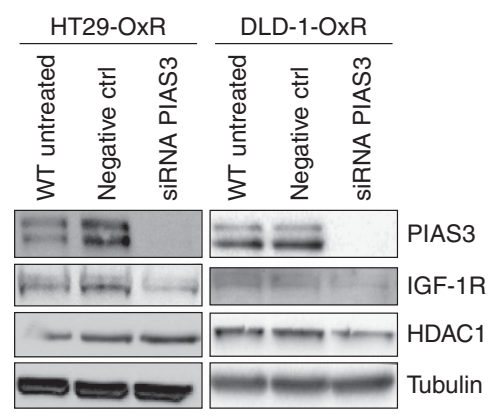

C

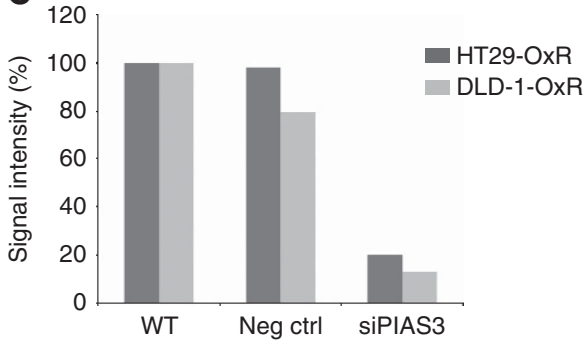

E

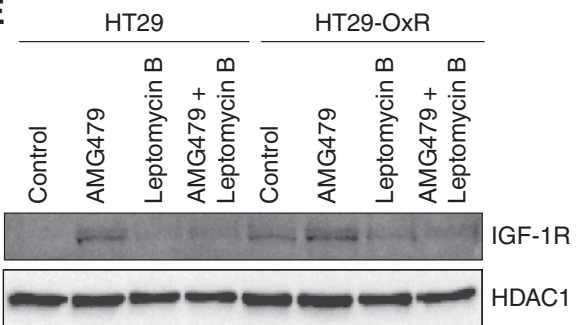

D

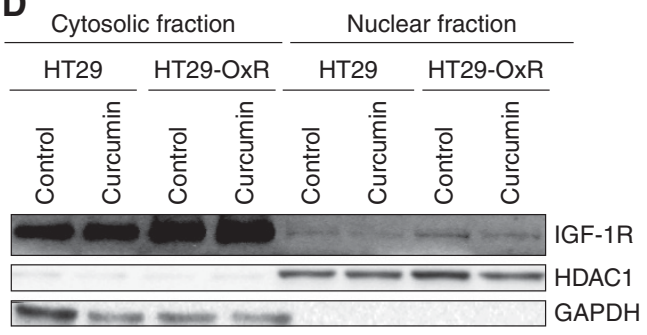

Figure 4. Loss-of-function of PIAS3 prevents sequestration of IGF-1R in the nuclear compartment of chemo-resistant cells. (A) Immunoblot analysis of PIAS3 after exposing the cells to ganitumab, NVP-AEW541, and the combination of both in HT29 and HT29-OxR cell lines. Note the correlation between the increase of PIAS3 and nuclear IGF-1R. HDAC1 was used as loading control. (B) Transient transfection of siRNAs against PIAS3 to HT29-OxR and DLD-1-OxR cell lines resulted in a significant knockdown of the target gene at $72 \mathrm{~h}$ post-transfection. Immunoblot analysis of IGF-1R revealed a consistent increase of the protein in the nuclear compartment. HDAC1 and tubulin were immunoblotted as a loading control. (C) Graph showing the quantification of the nuclear IGF-1R normalised to HDAC1 $72 \mathrm{~h}$ post-transfection with siRNAs against PIAS3. (D and E) HT29 and HT29-OxR cell lines were incubated for $24 \mathrm{~h}$ with curcumin and leptomycin B. Cytosolic and nuclear fractions were analysed by western blot with anti-IGF-1R antibody. Loading and fraction purity were controlled by reblotting the membrane with anti-HDAC1 and anti-GAPDH antibodies. Note that curcumin slightly decreases nuclear IGF-1R in HT29 and HT29-OxR cell lines.

\section{CONFLICT OF INTEREST}

The authors declare no conflict of interest.

\section{REFERENCES}

Aleksic T, Chitnis MM, Perestenko OV, Gao S, Thomas PH, Turner GD, Protheroe AS, Howarth M, Macaulay VM (2010) Type 1 insulin-like growth factor receptor translocates to the nucleus of human tumor cells. Cancer Res 70: 6412-6419.

Alonso-Espinaco V, Cuatrecasas M, Alonso V, Escudero P, Marmol M, Horndler C, Ortego J, Gallego R, Codony-Servat J, García-Albéniz X, Jares P, Castells A, Lozano JJ, Rosell R, Maurel J (2014) RAC1b overexpression correlates with poor prognosis in KRAS/BRAF WT metastatic colorectal cancer patients treated with first-line FOLFOX/ XELOX chemotherapy. Eur J Cancer 50: 1973-1981.

Alonso-Orduna V, Marmol M, Escudero P, Salud A, Safont MJ, Méndez JC, García-Girón C, Martín M, Fernandez-Martos C, García-Albéniz X, Feliu J, Maurel J (2014) 599PA validation of current prognostic scores in metastatic colorectal cancer (mCRC) and a new prognostic score (a GEMCAD Study). Ann Oncol 25: iv204-iv204.
Alonso V, Emperador PE, Urena MM, Gallego J, Rodriguez JR, Fernández J, Salud A, Falcó E, Manzano H, Zanui M, Gil M, Sarmiento UB, Martos CF, Calderero V, Ferrer AI, Cuatrecasas M, Rojo F, Feliu J, Maurel J, García-Albéniz X (2016) Prospective evaluation of BRAF, PI3K and PTEN as predictive and prognostic biomarkers in first-line advanced KRAS wildtype colorectal cancer treated with FOLFOX or FOLFIRI plus bi-weekly cetuximab. GEMCAD 10-02. Ann Oncol 27(Suppl 6): vil49-vi206.

Barras D, Missiaglia E, Wirapati P, Sieber OM, Jorissen RN, Love C, Molloy PL, Jones IT, McLaughlin S, Gibbs P, Guinney J, Simon IM, Roth AD, Bosman FT, Tejpar S, Delorenzi M (2017) BRAF V600E mutant colorectal cancer subtypes based on gene expression. Clin Cancer Res 23: 104-115.

Baserga R (1999) The IGF-I receptor in cancer research. Exp Cell Res 253: $1-6$.

Baserga R (2009) Customizing the targeting of IGF-1 receptor. Futur Oncol 5: 43-50.

Brenner H, Kloor M, Pox CP (2014) Colorectal cancer. Lancet 383: 1490-1502. Cohn AL, Tabernero J, Maurel J, Nowara E, Sastre J, Chuah BYS, Kopp M V, Sakaeva DD, Mitchell EP, Dubey S, Suzuki S, Hei Y-J, Galimi F, McCaffery I, Pan Y, Loberg R, Cottrell S, Choo S-P (2013) A randomized, placebocontrolled phase 2 study of ganitumab or conatumumab in combination with FOLFIRI for second-line treatment of mutant KRAS metastatic colorectal cancer. Ann Oncol Off J Eur Soc Med Oncol 24: 1777-1785.

Deng H, Lin Y, Badin M, Vasilcanu D, Strömberg T, Jernberg-Wiklund H, Sehat B, Larsson O (2011) Over-accumulation of nuclear IGF-1 receptor 
in tumor cells requires elevated expression of the receptor and the SUMOconjugating enzyme Ubc9. Biochem Biophys Res Commun 404: 667-671.

Feliu J, Salud A, Safont MJ, García-Girón C, Aparicio J, Vera R, Serra O, Casado E, Jorge M, Escudero P, Bosch C, Bohn U, Pérez-Carrión R, Carmona A, Martínez-Marín V, Maurel J (2014) First-line bevacizumab and capecitabine-oxaliplatin in elderly patients with mCRC: GEMCAD phase II BECOX study. Br J Cancer 111: 241-248.

Flanigan SA, Pitts TM, Eckhardt SG, Tentler JJ, Tan AC, Thorburn A, Leong S (2010) The insulin-like growth factor I receptor/insulin receptor tyrosine kinase inhibitor PQIP exhibits enhanced antitumor effects in combination with chemotherapy against colorectal cancer models. Clin Cancer Res 16: 5436-5446.

Girnita L, Girnita A, Brodin B, Xie Y, Nilsson G, Dricu A, Lundeberg J, Wejde J, Bartolazzi A, Wiman KG, Larsson O (2000) Increased expression of insulin-like growth factor I receptor in malignant cells expressing aberrant p53: functional impact. Cancer Res 60: 5278-5283.

Ii M, Li H, Adachi Y, Yamamoto H, Ohashi H, Taniguchi H, Arimura Y, Carbone DP, Imai K, Shinomura Y (2011) The efficacy of IGF-I receptor monoclonal antibody against human gastrointestinal carcinomas is independent of k-ras mutation status. Clin Cancer Res 17: 5048-5059.

Johnston CL, Cox HC, Gomm JJ, Coombes RC (1995) Fibroblast growth factor receptors (FGFRs) localize in different cellular compartments. A splice variant of FGFR-3 localizes to the nucleus. J Biol Chem 270: 30643-30650.

Kendziorra E, Ahlborn K, Spitzner M, Rave-Frank M, Emons G, Gaedcke J, Kramer F, Wolff HA, Becker H, Beissbarth T, Ebner R, Ghadimi BM, Pukrop T, Ried T, Grade M (2011) Silencing of the Wnt transcription factor TCF4 sensitizes colorectal cancer cells to (chemo-) radiotherapy. Carcinogenesis 32: 1824-1831.

Li C, Iida M, Dunn EF, Ghia AJ, Wheeler DL (2009) Nuclear EGFR contributes to acquired resistance to cetuximab. Oncogene 28: 3801-3813.

Lin SY, Makino K, Xia W, Matin A, Wen Y, Kwong KY, Bourguignon L, Hung MC (2001) Nuclear localisation of EGF receptor and its potential new role as a transcription factor. Nat Cell Biol 3: 802-808.

Maurel J, Martos CF, Richard MM, Alonso V, Méndez CM, Salud A, Pericay C, Aparicio J, Gallego J, Carmona A, Casado E, Manzano H, Horndler C, Rubini M, Cuatrecasas M, García-Albéniz X, Feliu J (2016) PULSE, a phase 2 study of mFOLFOX6-panitumumab (P) with biomarker stratification as firstline chemotherapy (CT), in patients (pts) with KRAS (exon 2) metastatic colorectal cancer (mCRC). A GEMCAD 09-03 study. Ann Oncol 27(Suppl 6): vil49-vi206.

Niu M, Wu S, Mao L, Yang Y (2013) CRM1 is a cellular target of curcumin: new insights for the myriad of biological effects of an ancient spice. Traffic 14: $1042-1052$

Packham S, Warsito D, Lin Y, Sadi S, Karlsson R, Sehat B, Larsson O (2014) Nuclear translocation of IGF-1R via p150(Glued) and an importin- $\beta$ / RanBP2-dependent pathway in cancer cells. Oncogene 34: 1-12.

Pollak M (2008) Insulin and insulin-like growth factor signalling in neoplasia. Nat Rev Cancer 8: 915-928.

Popovici V, Budinska E, Tejpar S, Weinrich S, Estrella H, Hodgson G, Van Cutsem E, Xie T, Bosman FT, Roth AD, Delorenzi M (2012) Identification of a poor-prognosis BRAF-mutant-like population of patients with colon cancer. J Clin Oncol 30: 1288-1295.

Ritterhoff T, Das H, Hofhaus G, Schröder RR, Flotho A, Melchior F (2016) The RanBP2/RanGAP1 ${ }^{*}$ SUMO1/Ubc9 SUMO E3 ligase is a disassembly machine for Crm1-dependent nuclear export complexes. Nat Commun 7: 11482.

Salani B, Passalacqua M, Maffioli S, Briatore L, Hamoudane M, Contini P, Cordera R, Maggi D (2010) IGF-IR internalizes with caveolin-1 and PTRF/Cavin in Hacat cells. PLoS One 5: e14157.
Saltz LB, Clarke S, Díaz-Rubio E, Scheithauer W, Figer A, Wong R, Koski S, Lichinitser M, Yang T-S, Rivera F, Couture F, Sirzén F, Cassidy J (2008) Bevacizumab in combination with oxaliplatin-based chemotherapy as first-line therapy in metastatic colorectal cancer: a randomized phase III study. J Clin Oncol 26: 2013-2019.

Saydmohammed M, Joseph D, Syed V (2010) Curcumin suppresses constitutive activation of STAT-3 by up-regulating protein inhibitor of activated STAT-3 (PIAS-3) in ovarian and endometrial cancer cells. J Cell Biochem 110: 447-456.

Sehat B, Andersson S, Vasilcanu R, Girnita L, Larsson O (2007) Role of ubiquitination in IGF-1 receptor signaling and degradation. PLoS One 2: e340.

Sehat B, Tofigh A, Lin Y, Trocmé E, Liljedahl U, Lagergren J, Larsson O (2010) SUMOylation mediates the nuclear translocation and signaling of the IGF-1 receptor. Sci Signal 3: ra10.

Sundvall M, Korhonen A, Vaparanta K, Anckar J, Halkilahti K, Salah Z, Aqeilan RI, Palvimo JJ, Sistonen L, Elenius K (2012) Protein inhibitor of activated STAT3 (PIAS3) protein promotes SUMOylation and nuclear sequestration of the intracellular domain of ErbB4 protein. J Biol Chem 287: 23216-23226.

Van Cutsem E, Eng C, Nowara E, Swieboda-Sadlej A, Tebbutt NC, Mitchell E, Davidenko I, Stephenson J, Elez E, Prenen H, Deng H, Tang R, McCaffery I, Oliner KS, Chen L, Gansert J, Loh E, Smethurst D, Tabernero J (2014) Randomized phase Ib/II trial of rilotumumab or ganitumab with panitumumab versus panitumumab alone in patients with wild-type KRAS metastatic colorectal cancer. Clin Cancer Res 20: 4240-4250.

Van Cutsem E, Köhne C-H, Láng I, Folprecht G, Nowacki MP, Cascinu S, Shchepotin I, Maurel J, Cunningham D, Tejpar S, Schlichting M, Zubel A, Celik I, Rougier P, Ciardiello F (2011) Cetuximab plus irinotecan, fluorouracil, and leucovorin as first-line treatment for metastatic colorectal cancer: updated analysis of overall survival according to tumor KRAS and BRAF mutation status. J Clin Oncol 29: 2011-2019.

Vecchione L, Gambino V, Raaijmakers J, Schlicker A, Fumagalli A, Russo M, Villanueva A, Beerling E, Bartolini A, Mollevi DG, El-Murr N, Chiron M, Calvet L, Nicolazzi C, Combeau C, Henry C, Simon IM, Tian S, in 't Veld S, D'ario G, Mainardi S, Beijersbergen RL, Lieftink C, Linn S, Rumpf-Kienzl C, Delorenzi M, Wessels L, Salazar R, Di Nicolantonio F, Bardelli A, van Rheenen J, Medema RH, Tejpar S, Bernards R (2016) A vulnerability of a subset of colon cancers with potential clinical utility. Cell $\mathbf{1 6 5}$ : 317-330.

Wang S-C, Lien H-C, Xia W, Chen I-F, Lo H-W, Wang Z, Ali-Seyed M, Lee D-F, Bartholomeusz G, Ou-Yang F, Giri DK, Hung M-C (2004) Binding at and transactivation of the COX-2 promoter by nuclear tyrosine kinase receptor ErbB-2. Cancer Cell 6: 251-261.

Warsito D, Sjöström S, Andersson S, Larsson O, Sehat B (2012) Nuclear IGF1R is a transcriptional co-activator of LEF1/TCF. EMBO Rep 13: 244-250.

Werner H, LeRoith D (1996) The role of the insulin-like growth factor system in human cancer. Adv Cancer Res 68: 183-223.

This work is published under the standard license to publish agreement. After 12 months the work will become freely available and the license terms will switch to a Creative Commons AttributionNonCommercial-Share Alike 4.0 Unported License.

Supplementary Information accompanies this paper on British Journal of Cancer website (http://www.nature.com/bjc) 